\title{
Perfil epidemiológico dos pacientes atendidos em uma unidade de terapia intensiva cardiopulmonar de um hospital de referência do Ceará
}

\section{Profile epidemiological of patients carried out in a cardiopulmonary intensive therapy unit of a reference hospital of Ceará}

Fernanda Lúcia Oliveira da Silva Barros ${ }^{1}$ Karine Maria Martins Bezerra Carvalho². Antonia Rayza Negreiros Falcão ${ }^{3,4}$. José Diógenes Marques Ribeiro Filho ${ }^{3}$. Raimunda Rosilene Magalhaes Gadelha ${ }^{4}$. Dyely de Carvalho Oliveira Campos ${ }^{4}$.

1 Faculdade do Vale do Jaguaribe (FVJ), Aracati, Ceará, Brasil. 2 Faculdade Estácio FIC, Fortaleza, Ceará, Brasil. 3 Hospital Dr Carlos Alberto Studart Gomes, Fortaleza, Ceará, Brasil. 4. Centro Universitário Católica de Quixadá (Unicatólica), Quixadá, Ceará, Brasil.

\section{RESUMO}

A identificação do perfil epidemiológico de uma Unidade de Terapia Intensiva é fundamental no auxílio de tomada de decisões e na criação de estratégias visando o aperfeiçoamento da qualidade do serviço. Objetivo: identificar o perfil dos pacientes internados na Unidade Cardiopulmonar. Metodologia: estudo caráter descritivo retrospectivo, com corte transversal e abordagem quantitativa, realizado em um hospital de referência do Ceará. Foram analisados os dados de prontuários de janeiro a julho de 2017. Resultados: os resultados evidenciaram que a população é predominantemente do sexo masculino, idosa e procedente na maior parte da emergência, vindo também do Centro de Terapia Intensiva Pós Adulto, enfermarias, Centro Cirúrgico, Unidade Semi-intensiva e da Hemodinâmica. O tempo médio de internação foi de 14 dias. Os diagnósticos de internação mais encontrados foram pneumonia, seguida de insuficiência cardíaca congestiva, pós-operatório de troca valvar, doença pulmonar obstrutiva crônica, sepse, edema agudo pulmonar e infarto agudo do miocárdio sem supra desnivelamento do segmento ST. No período da pesquisa ocorreram mais transferências do que óbitos. O índice de óbito foi de 39,80\%. Conclusão: o conhecimento desses dados é fundamental para otimizar o processo de trabalho e de cuidado, possibilitando melhor planejar ações de cuidado em saúde.

Palavras-chave: Unidades de Terapia Intensiva. Perfil epidemiológico. Sistema Único de Saúde.

\section{ABSTRACT}

The identification of the epidemiological profile of an ICU is fundamental in the aid of decision making and in the creation of strategies aiming at the improvement of the quality of the service. Objective: to identify the profile of patients admitted to the Cardiopulmonary Unit. Methodology: this is a retrospective, descriptive character study with cross section, and presents a quantitative approach, carried out in a referral hospital of Ceará. Being parsed the data through charts from January to July 2017 that filled a semi structured form directing the search for the findings of relevance to the study. Results: the results showed that the population is predominantly male, older and resident in most of the emergency, coming also from the Center Post Adult intensive care, hospital wards, operating room, intensive care unit and the Hemodynamics. The average hospital stay was 14 days. The most common diagnoses of hospitalization were pneumonia, followed by congestive heart failure, postoperative valve replacement, chronic obstructive pulmonary disease, sepsis, acute pulmonary edema and acute myocardial infarction without ST segment e depression above, during the period of Research occurred more transfers than deaths. The death index was 39,80\%. Conclusion: the knowledge of this data is essential to optimize the process of work and care, making it possible to better plan health care actions.

Keywords: Intensive care units. Epidemiological profile. Unified Health System.

Autor correspondente: Karine Maria Martins Bezerra Carvalho, Rua Fiúza de Pontes, 299, Centro, Fortaleza, Ceará, Brasil. CEP: $60140-170$. Telefone: +55 85 99914-0579. E-mail: karinecarvalho@unicatolicaquixada.edu.br

Conflito de interesses: Não há qualquer conflito de interesses por parte de qualquer um dos autores.

Recebido em: 26 Set 2018; Revisado em: 06 Mai 2019; Aceito em: 30 Set 2019. 


\section{INTRODUÇÃO}

A epidemiologia é o estudo do comportamento das doenças em uma determinada população, com intuito de proporcionar novos conhecimentos sobre esse grupo e gerar transformação nas condições de vida e saúde dos indivíduos que a compõe. ${ }^{1}$

As investigações epidemiológicas podem contribuir para melhoria do atendimento, organização dos serviços e adequação dos protocolos de conduta. Diante do perfil epidemiológico de uma região, se determina qual nível de atenção terá mais ênfase para essa população: primária, secundária e/ou terciária. ${ }^{2}$

Um ambiente de cuidado abrange um complexo contexto que envolve várias variáveis, dentre elas as relacionadas ao ambiente, a equipe multidisciplinar, aos cuidados prestados, tecnologia disponível e recurso para intervenções, se necessárias. ${ }^{3}$ A estrutura de uma unidade de terapia intensiva (UTI), como hoje é conhecida, foi desenvolvida nos anos 1950, em resposta à epidemia de poliomielite. Neste período, destacava-se o suporte invasivo de ventilação mecânica realizado por pressão negativa através de aparelhos denominados "pulmão de aço". ${ }^{4}$

UTI é um ambiente que tem suporte necessário para pacientes graves e instáveis que precisam de uma alta complexidade de atenção por ter um potencial risco de vida. ${ }^{5}$ Utilizam-se recursos para diminuir o risco de complicações pós-operatórias, como a ventilação por pressão positiva não invasiva, dentre outros recursos que sejam viáveis para o bem-estar do paciente. ${ }^{6}$

A partir das informações supracitadas surgiu a necessidade de determinar o perfil epidemiológico de uma UTI com assistência para pessoas com doenças cardiopulmonares situada em um hospital de alta complexidade do estado do Ceará.

Pretende-se com este trabalho caracterizar as patologias mais encontradas, verificar o tempo médio de internação, avaliar origem e desfechos, além do perfil sociodemográfico (idade, sexo) dos pacientes. Por meio desse esclarecimento, espera-se contribuir com o meio científico e através do conhecimento do perfil epidemiológico da unidade de terapia intensiva cardiopulmonar possibilitando a equipe uma otimização de sua atuação e o desenvolvimento de habilidades e estratégias voltadas para sua maior demanda.

\section{METODOLOGIA}

O estudo tem um caráter descritivo retrospectivo, com corte transversal, e apresenta abordagem quantitativa.

O método quantitativo tem como diferença do qualitativo a questão de quantificar dados fundamentados em amostras representativas, realizando assim uma análise estatística. ${ }^{7}$

A pesquisa com caráter descritivo busca identificar, analisar e/ ou registrar fatos que venham gerando mudanças sejam elas fisiológicas, patológicas dentre outras. Nesse estudo, após a coleta de dados é realizada uma análise em relação às variáveis encontradas. $^{8}$

A pesquisa retrospectiva explora fatos do passado, podendo ser delineado para retornar, ou seja, do momento atual até um determinado ponto no passado, há vários anos, onde o pesquisador pode marcar um ponto no passado e conduzir a pesquisa até o momento presente pela análise documental. ${ }^{9}$

Este estudo foi realizado no Hospital de Messejana, Av. Frei Cirilo $n^{0}: 3480$, CEP: 60840-285, Fortaleza-CE, na Unidade de Terapia Intensiva Cardiopulmonar. A unidade de terapia intensiva cardiopulmonar (UCP), onde foi desenvolvida esta pesquisa, possui 7 leitos destinados a pacientes adultos, tem um caráter clínico e uma equipe multiprofissional envolvida no atendimento e na busca da recuperação e/ou diminuição de agravos desses pacientes.

A população dessa pesquisa foi composta por pacientes da Unidade de Terapia Intensiva do Hospital de Messejana. A amostra foi composta pelos pacientes que foram internados no primeiro semestre de 2017. Foram excluídos os prontuários com dados incompletos.

O presente artigo utilizou como coleta de dados os prontuários de pacientes internados na UCP no período de janeiro a julho de 2017. Como instrumento para coleta foi utilizado um formulário que foi devidamente preenchido de acordo com os prontuários existentes na unidade. $\mathrm{O}$ formulário obtém as seguintes variáveis: 1) dados pessoais (idade, cor, gênero) e 2) patologia, tempo de internação, desfecho, origem, óbitos (gênero e patologias mais recorrentes). Os dados só foram coletados após assinatura da carta de anuência pela direção coparticipante autorizando a coleta de dados, juntamente com a aprovação no CEP do referido hospital no período de abril a junho de 2018.

Os dados foram tabulados, organizados em tabelas feitas no Microsoft Excel 2013, quantificados em porcentagens e transformados em gráficos. Analisados através dos resultados obtidos.

Os aspectos éticos em uma pesquisa que envolve seres humanos garantem confidencialidade, não utilização das informações disponibilizadas para prejuízo dos indivíduos, bem como emprego dessas informações apenas para os fins previstos na pesquisa.

Este trabalho foi aprovado pelo Comitê de Ética em Pesquisa (CEP) do Hospital de Messejana Dr Carlos Alberto Studart Gomes, respeitando os princípios éticos presentes na Resolução no 466/12 do Conselho Nacional de Saúde (CNS) do Ministério de Saúde (MS), relativos à pesquisa com seres humanos, sob parecer $n^{\circ} 2.564 .979$.

\section{RESULTADOS}

De acordo com o levantamento obtido nos prontuários, no período estimado pela pesquisa, receberam atendimento da 
equipe multidisciplinar da Unidade de Terapia Cardiopulmonar (UCP) do Hospital de Messejana 150 pessoas, dessas, 7 foram excluídas do estudo devido possuir dados incompletos nas fichas analisadas. A média de idade encontrada foi de 62,67 anos, 92 $(64,33 \%)$ eram idosos, ou seja, tinham idade acima de 60 anos.

Além disso, 74 (51,74\%) correspondiam ao gênero masculino, e $69(48,25 \%)$ ao feminino, o que resulta na prevalência de homens (Tabela 01).

A média dos dias de internação dos pacientes foi de 14,57 dias, variando entre 1 e no máximo 63 dias. Quanto ao setor de origem dos pacientes admitidos nesse período, os resultados demonstraram: 72,71\% (104) foram provenientes da emergência; 14,68\% (21) do Centro de Terapia Intensiva Pós Adulto (CETI); 9,76\% (14) das enfermarias; 1,39\% (2) do Centro Cirúrgico (CC.); 0,69\% (1) da Unidade Semi-intensiva e $0,69 \%$ (1) da Hemodinâmica (Tabela 02).

Tabela 1. Perfil dos pacientes da UCP quanto ao gênero.

\begin{tabular}{lll}
\hline Gênero & Número de pacientes & \% \\
\hline Masculino & 74 & 51,74 \\
Feminino & 69 & 48,25 \\
\hline
\end{tabular}

Tabela 2. Setor de origem dos pacientes atendidos na UCP.

\begin{tabular}{lll}
\hline Proveniência & Número de pacientes & $\mathbf{\%}$ \\
\hline Emergência & 104 & 72,72 \\
CETI & 21 & 14,68 \\
Unidades de Enfermaria & 14 & 9,79 \\
Centro cirúrgico & 2 & 1,39 \\
Unidade Semi Intensiva & 1 & 0,69 \\
Hemodinâmica & 1 & 0,69 \\
\hline
\end{tabular}

Dentre os diagnósticos de internação mais encontrados desse estudo, os que mais foram citados são: pneumonia (a patologia mais recorrente), insuficiência cardíaca congestiva, pós-operatório de troca valvar, doença pulmonar obstrutiva crônica (DPOC), sepse, edema agudo pulmonar e infarto agudo do miocárdio sem supra desnivelamento do segmento ST. (Tabela 03). Vale lembrar que alguns pacientes tinham mais de um problema de saúde e que estas também foram as principais causas de óbitos na unidade.

No período estipulado da pesquisa, foram realizadas 86 transferências sendo elas respectivamente para: Unidades (Enfermarias), 61 (42,54\%); Unidade Semi intensiva, 22 (15,38\%); Centro Cirúrgico, 2 (1,39\%) e UTI Respiratória, $1(0,69 \%)$. Além dos 57 óbitos que corresponderam a 39,8\% (Tabela 04).

O gênero mais prevalente nos óbitos foi o masculino com 37 $(64,90 \%)$. Os pacientes tinham média de idade 66,36 anos, as idades variavam de 32 a 92 anos (Tabela 05).
Tabela 3. Patologias mais encontradas nos pacientes da UCP.

\begin{tabular}{lll}
\hline Causa da admissão na UCP & $\begin{array}{l}\text { Número de } \\
\text { pacientes }\end{array}$ & $\mathbf{\%}$ \\
\hline Pneumonia & 43 & 20,06 \\
Insuficiência Cardíaca Congestiva & 41 & 28,67 \\
Insuficiência Respiratória Aguda & 25 & 17,48 \\
Pós Operatório Troca Valvar & 20 & 13,98 \\
Doença Pulmonar Obstrutiva Crônica & 19 & 13,28 \\
Sepse & 18 & 12,58 \\
Edema Agudo Pulmonar & 15 & 10,48 \\
Infarto Agudo do Miocárdio sem supra & 12 & 8,39 \\
desnivelamento do segmento ST & & \\
\hline
\end{tabular}

*um paciente pode possuir mais de uma patologia.

Tabela 4. Destino de encaminhamento dos pacientes da UCP.

\begin{tabular}{lll}
\hline Transferência & Número de pacientes & $\mathbf{\%}$ \\
\hline Unidades & 61 & 42,54 \\
Óbitos & 57 & 39,80 \\
Semi intensiva & 22 & 15,38 \\
Centro Cirúrgico & 2 & 1,39 \\
UTI Respiratória & 1 & 0,69 \\
\hline
\end{tabular}

Tabela 5. Gênero dos óbitos ocorridos nos pacientes da UCP.

\begin{tabular}{lll}
\hline Sexo & Número de pacientes & $\%$ \\
\hline Masculino & 37 & 64,90 \\
Feminino & 20 & 35,80 \\
\hline
\end{tabular}

\section{DISCUSSÃO}

A identificação do perfil epidemiológico de uma UTI é fundamental no auxílio de tomada de decisões e na criação de estratégias visando o aperfeiçoamento da qualidade do serviço.

Inicialmente observou-se que a maioria dos pacientes atendidos na UTI cardiopulmonar tinham idade acima de 60 anos. A elevada faixa etária observada em pacientes de UTI geralmente se deve ao fato de as alterações fisiológicas do envelhecimento favorecerem a necessidade de procedimentos invasivos. ${ }^{10}$

Além disso, o estilo de vida adotado deixa os indivíduos expostos a condicionantes e determinantes de saúde. De acordo com os hábitos adquiridos, distúrbios e incapacidades podem se desenvolver. Hábitos como sedentarismo, fumo, uso abusivo de álcool, alimentação inadequada, podem desencadear doenças como hipertensão arterial, diabetes, cardiopatias, doenças pulmonares crônicas, entre outras. Aliado aos hábitos adquiridos por cada indivíduo está a mudança do perfil epidemiológico brasileiro. O envelhecimento da população favoreceu o aumento das doenças crônico degenerativas em detrimento das infectocontagiosas. ${ }^{11}$ 
Corroborando com os resultados encontrados, um estudo sobre perfil dos pacientes internados na unidade de terapia intensiva adulto de um hospital universitário constatou que a idade média dos internados foi de 64,8 anos $( \pm 5,65)$. Dentre todos os admitidos, 49 (47\%) eram idosos. ${ }^{12}$

Quanto ao gênero, observou-se um maior número de internações de indivíduos do gênero masculino. Esse dado está de acordo com diversos estudos avaliando o perfil de pacientes admitidos em UTIs no Brasil. ${ }^{12-14}$

A saúde dos homens é um assunto complexo. Além dos fatores biológicos, fatores sociais e comportamentais relacionados ao gênero masculino aumentam os riscos de adoecimento e morte entre os homens. O homem é encorajado a mostrar sempre força e invulnerabilidade. Estudos mostram que esse conceito de masculinidade hegemônica tem influenciado nas taxas de mortalidade masculina. ${ }^{15}$

A maioria dos pacientes da UCP deram entrada na emergência, demonstrando a dinâmica do hospital. A prioridade é receber os pacientes que deram entrada na emergência e tiveram necessidade de cuidados intensivos, sendo encaminhados para a Sala de Parada Cardiorrespiratória (S.P.C.R.).

Sabe-se que o Brasil enfrenta um sério problema relacionado a carência de leitos de terapia intensiva. Esse fato aliado a gravidade dos casos admitidos, prolongam o tempo de permanência e os custos. A média de tempo de internação foi de aproximadamente quatorze dias na UTI estudada, porém, houve uma variação de um a sessenta e três dias. Esses dados também corroboram com os dados nacionais relacionados a unidades de terapia intensiva.

O tempo de internação pode variar muito, dependendo do tipo da doença de base, da gravidade, das complicações, podendo assim o indivíduo permanecer apenas 24 horas ou até meses internado. ${ }^{16}$

Um estudo traçando o perfil de pacientes atendidos num hospital Universitário de uma cidade do interior do Rio Grande do Sul evidenciou um tempo médio de internação de 14 dias, o tempo de maior permanência registrado foi de 95 dias. ${ }^{12}$

As causas da internação mais prevalentes foram pneumonia, insuficiência cardíaca congestiva, pós-operatório de troca valvar, doença pulmonar obstrutiva crônica (DPOC), sepse, edema agudo pulmonar e infarto agudo do miocárdio sem supra desnivelamento do segmento ST. Dados justificados pelo fato do Hospital ser especializado em tratamento de doenças cardíacas e pulmonares, além da alta incidência no âmbito nacional destas.

Os avanços tecnológicos da assistência a saúde nas últimas décadas têm elevado a sobrevida dos pacientes críticos. Associado a esse prolongamento da vida está o aumento dos riscos relacionados com a internação. Dentre as infecções hospitalares, destaca-se a pneumonia associada a ventilação mecânica (PAV), definida como uma pneumonia que surge entre 48 e 72 horas após a intubação ou até 48 horas após extubação. Sua incidência varia de 9 a $40 \%$ das infecções hospitalares e é responsável por 20 a $60 \%$ da mortalidade nas UTIs. Além disso, a alta incidência de PAV prolonga as internações e aumenta os custos. ${ }^{17}$

Um estudo com 20 pacientes ventilados mecanicamente hospitalizados na UTI do Hospital Nossa Senhora da Conceição (HNSC), localizado em Tubarão - SC, entre fevereiro e agosto de 2013, chegou ao resultado de que a incidência da PAVM foi de $31,8 \%$, e a mortalidade de $51,7 \%{ }^{18}$

O presente estudo demonstrou uma alta incidência de pneumonia na UTI estudada. Embora os prontuários não tenham diferenciado qual o tipo de pneumonia entre PAV e PAC (pneumonia adquirida na comunidade), o fato de a maioria dos pacientes internados em UTIs necessitarem de suporte ventilatório, sinaliza para uma alta incidência de PAV. Esses dados foram considerados muito relevantes pois demonstram a necessidade de melhorar os protocolos de prevenção de PAV utilizados nesta UTI. A educação da equipe de saúde, vigilância de PAV e microbiológica, prevenção de fatores de risco associados ao tratamento e prevenção da transmissão de micro-organismos são medidas recomendadas que deverão ser revistas na UTI estudada.

Um estudo realizado na UTI cardiopulmonar de uma instituição pública localizada em Fortaleza-CE, mostrou que as principais causas de indicação para a UTI cardiopulmonar foram respectivamente: infarto agudo do miocárdio (IAM) com 166 pacientes; insuficiência cardíaca congestiva (ICC), 61; valvulopatia, 22; angina, 17; miocardiopatia dilatada, 17; doença pulmonar obstrutiva crônica, 9; cor pulmonale, 9; estenose traqueal, 3; e pacientes em pós-operatório. ${ }^{19}$

Os dados mostraram que 39,8\% dos pacientes foram a óbito. Sabe-se que a gravidade com que o paciente chega à UTI tem influência nos preditores de mortalidade. Assim, em estudos posteriores, serão incluídos no perfil dos pacientes admitidos nesta UTI escores de mortalidade como o APACHE II. O alto número de óbitos se dá, em sua maioria, devido os pacientes encaminhados para UCP na maior parte serem portadores de doenças crônicas que desencadeiam patologias mais severas, ou seja, além de doenças de base o paciente ainda desenvolve uma "cascata" de problemas sistêmicos.

Uma pesquisa com o objetivo de identificar fatores de risco em idosos admitidos em UTIs foi realizada através de um estudo de coorte retrospectivo coletando dados de 457 prontuários de idosos internados numa UTI na cidade de Natal-RN, Brasil. Os resultados demonstraram que os fatores prognósticos de origem demográfica, clínica, referentes ao tipo de internação e à rede de serviços de saúde, possui grande impacto relativo a fatores de riscos na vida dos idosos, ou seja, para os autores, a idade avançada e a qualidade de vida interferem no prognóstico. ${ }^{20}$

Outro estudo com o objetivo de investigar fatores associados ao óbito em pacientes idosos observou, num total de 84 pacientes internados em UTI, uma média de idade de $73 \pm 7,6$ anos, $59 \%$ do gênero feminino, e uma mortalidade de $62,8 \%$, ou seja, o dobro de óbitos encontrados na presente pesquisa. ${ }^{21}$

Nessa pesquisa, os pacientes foram a óbito por mais de uma 
causa, sendo as mais recorrentes respectivamente: pneumonia, insuficiência cardíaca congestiva, insuficiência respiratória aguda, sepse, infarto agudo do miocárdio sem supra desnivelamento do segmento ST, edema agudo pulmonar e pós-operatório tardio de troca valvar.

Conclui-se que os pacientes internados na Unidade de Terapia Intensiva Cardiopulmonar em estudo, caracterizam-se pela predominância do sexo masculino e idosos, com média de dias de internação de 14 dias, tendo como patologias mais recorrentes: pneumonia, insuficiência cardíaca congestiva, insuficiência respiratória aguda, pós-operatório de troca valvar, DPOC, sepse, dentre outras. O alto índice de recorrência de pneumonia indica a necessidade de se reduzir esse fator, através de estratégias que deverão ser planejadas e executadas no ambiente em prol de uma diminuição de casos.

\section{REFERÊNCIAS}

1. Carvalho CA, Pinho JR, Garcia PT. Epidemiologia: conceitos e aplicabilidade no sistema único de saúde. São Luís: EDUFMA; 2017.

2. Rothman KJ, Greenland S, Lash TL. Epidemiologia moderna. 3.ed. [place unknown]: ARTMED; 2016.

3. Haino B, Marlus V. Auditoria em Saúde. [place unknown]: Saraiva; 2017. (Série Gestão Estratégica de Saúde).

4. Fernandes HS, Pulzi SA Júnior, Costa R Filho. Qualidade em terapia intensiva. Rev Bras Clin Med. 2010;8:37-45.

5. Ehlers VJ, Watson H, Moleki MM. Factors contributing to sleep deprivation in a multi-disciplinary intensive care unit in South Africa. Curationis. 2013;36(1):1-8.

6. Mateos-Pañero B, Sánchez-Casado M, Castaño-Moreira B, Paredes-Astillero I, López-Almodóvar LF, Bustos-Molina F. Valoración del Euroscore y SAPS III como predictor de mortalidade hospitalaria em el postoperatorio de cirugía cardíaca. Rev Esp Anestesiol Reanim. 2017;64(5):273-81.

7. Linhares BF, Alves DS. Metodologia de ensino em pesquisa social quantitativa. Rev Pensamento Plural. 2014;14:23-39.

8. Perovano DG. Manual de metodologia científica. Paraná: Juruá; 2014.

9. Giraldi LP, Sigolo SR. Perspectiva longitudinal de pesquisa em educação no Brasil. Atos de Pesquisa em Educação. 2016;11(1):2-22.

10. Moreira ET, Nunes TF, Santos ES, Calles AC. Perfil e gravidade dos pacientes admitidos em unidades de terapia intensiva: uma revisão de literatura. Cadernos de Graduação - Ciências Biológicas e da Saúde Fits. 2013;1(2):45-52.

11. Castro RR, Barbosa N, Alves T, Najberge E. Perfil das internações em unidades de terapia intensiva adulto na cidade de ANÁPOLIS GOIÁS - 2012. Revista de Gestão em Sistemas de Saúde - RGSS. 2016;5(2):115-24.
As tecnologias nas unidades de tratamento intensivo são tão importantes quanto o conhecimento de dados epidemiológicos da população atendida, já que é essencial a caracterização dos pacientes para auxiliar nas admissões e altas devido ao favorecimento de critérios objetivos para essas finalidades, além de contribuir para o uso de leitos de forma mais organizada e racional, evitando assim expor o paciente a riscos. Ou seja, essa pesquisa proporcionará à equipe uma otimização de sua atuação e o desenvolvimento de habilidades e estratégias voltadas para sua maior demanda.

Contudo, sugerimos o desenvolvimento de estudos semelhantes, com o intuito de buscar ampliar dados sobre a caracterização dos pacientes e o impacto que isso pode gerar na assistência aos mesmos.

12. Favarin SS, Camponogara S. Perfil dos pacientes internados na Unidade de Terapia Intensiva adulto de um Hospital Universitário. Rev Enferm UFSM. 2012;2(2):320-9.

13. Cravo CD, Miranzi SS, Iwamoto HH, Souza JL Júnior. Perfil epidemiológico dos pacientes em hemodiálise de um Hospital Universitário. Cienc Cuid Saude. 2011;10(1):110-5.

14. Nogueira PR, Rassi S, Corrêa KS. Perfil epidemiológico, clínico e terapêutico da insuficiência cardíaca em hospital terciário. Arq Bras Cardiol. 2010;95(3):392-8.

15. Fraga JC, Corrêa AC, Rocha RM, Silva LA, Medeiros RM, Mozer IT. Principais causas da mortalidade masculina e os anos potenciais de vida perdidos por estes agravos. Revista Cienc Cuid Saude. 2016;15(4):746-54.

16. Carvalho MP, Barrozo AF. Mobilização precoce no paciente crítico internado em unidade de terapia intensiva. Braz J Surg Clin. 2014;8(3):66-71.

17. Ribeiro KR, Anjos EG, Oliveira EM. Enfermagem em ventilação mecânica: cuidados na prevenção de pneumonia. Revista Recien. 2016;6(16):57-71.

18. Kock KS, Rosa BC, Martignago NN, Maurici R. Pneumonia associada à ventilação mecânica (PAVM): incidência e desfecho clínico em uma Unidade de Terapia Intensiva no Sul de Santa Catarina. Arq Catarin Med. 2017;46(1):2-11.

19. Rocha MS, Caetano JA, Soares E, Medeiros FG. Caracterização da população atendida em unidade de terapia intensiva: subsídio para a assistência. Rev Enferm UERJ. 2007;15(3):411-6.

20. Bonfada D, Santos MM, Lima KC, Garcia-Altés A. Análise de sobrevida de idosos internados em Unidades de Terapia Intensiva. Rev Bras Geriatr Gerontol. 2017;20(2):198-206.

21. Alves GC, Silva GB Júnior, Lima RS, Sobral JB, Mota RM, Abreu $\mathrm{KL}$, et al. Fatores de risco para óbito em pacientes idosos gravemente enfermos. Rev Bras Ter Intensiva. 2010;2(2):138-43.

\section{Como citar:}

Barros FL, Carvalho KM, Falcão AR, Ribeiro JD Filho, Gadelha RR, Campos DC. Perfil epidemiológico dos pacientes atendidos em uma unidade de terapia intensiva cardiopulmonar de um hospital de referência do Ceará. Rev Med UFC. 2020 abr-jun;60(2):26-30. 